\title{
High peak power and sub-picosecond Fourier-limited pulse generation from passively mode-locked monolithic two- section gain-guided tapered InGaAs Quantum-Dot lasers
}

\author{
D. I. Nikitichev, ${ }^{1,{ }^{\star}}$ Y. Ding, ${ }^{1}$ M. A. Cataluna, ${ }^{1}$ E. U. Rafailov, ${ }^{1}$ L. Drzewietzki, ${ }^{2}$ S. Breuer, ${ }^{2}$ W. Elsaesser, ${ }^{2}$ M. \\ Rossetti, ${ }^{3}$ P. Bardella, ${ }^{3}$ T. Xu, ${ }^{3}$ I. Montrosset, ${ }^{3}$ I. Krestnikov, ${ }^{4}$ D. Livshits, ${ }^{4}$ M. Ruiz, ${ }^{5}$ M. Tran, ${ }^{5}$ Y.Robert, ${ }^{5}$ M. \\ Krakowski ${ }^{5}$ \\ ${ }^{1}$ University of Dundee, School of Engineering, Physics and Mathematics, Dundee, DD1 4HN, UK \\ 2 Institut fur Angewandte Physik, Technische Universitat Darmstadt, Schlo gartenstr. 7, 64289 Darmstadt, Germany \\ ${ }^{3}$ Politecnico di Torino, Dipartimento di Elettronica, Turin, Italy \\ 4 Innolume GmbH, Konrad-Adenauer-Allee 11, 44263 Dortmund, Germany \\ 5 III-V Lab, 1 Av Augustin Fresnel, Campus de Polytechnique, 91767 Palaiseau, France
}

Abstract: We report on the development of a new generation of high-power ultrashort pulse quantum-dot lasers with tapered gain section. Two device designs are proposed and fabricated, with different total lengths and absorber-to-gain-section lengthratios. These designs have been informed by numerical simulations of the dynamic mode-locking regimes and their dependence on the structural parameters. One device design demonstrated a record-high peak power of $17.7 \mathrm{~W}$ with 1.26 ps pulse width and a second design enabled the generation of a Fourier-limited $672 \mathrm{fs}$ pulse width with a peak power of $3.8 \mathrm{~W}$. A maximum output average power of $288 \mathrm{~mW}$ with $28.7 \mathrm{pJ}$ pulse energy was also attained. In addition, the integrated timing jitter of $2.6 \mathrm{ps}$ and farfield patterns are demonstrated.

\section{Introduction}

Low-cost, miniature semiconductor diode lasers are highly efficient light sources which have been widely used in a range of applications, most notably in optical fiber communications and biomedical applications. The improvement in their performance in terms of ultrashort pulse generation, good beam quality, high peak power as well as high average power will transform such devices into extraordinarily interesting and promising candidates for an extended variety of applications in industry and science, ranging from nonlinear biomedical imaging, nano-surgery, materials processing through to laser projector displays and free space optical communications [1-5]. Quantum-well tapered lasers with index-guided straight ridge waveguide section coupled to gain-guided tapered section were effectively realized, generating high output power (more than $14 \mathrm{~W}$ at $980 \mathrm{~nm}$ ) with good beam quality $\left(\mathrm{M}^{2}<2\right)[6-8]$. Mode-locking is one of the most effective techniques for producing ultrashort pulses from semiconductors lasers. The combination of tapered waveguide laser design for higher power and passively mode-locking technique for short pulse duration has been successfully shown by Mar et al. [9]. In that paper, $2 \mathrm{~W}$ peak power with 3.3-ps pulse duration was demonstrated in a passively mode-locked tapered laser (with $\ln _{0.2} \mathrm{Ga}_{0.8}$ As quantum well active region) using external cavity feedback [9]. Some other high power mode-locked technologies can be used, for example: vertical external cavity surface emitting lasers (VECSELs) [10] or slab-coupled optical waveguide lasers (SCOWLs) [11]. For instance, a 1.5 $\mu \mathrm{m}$ InGaAsP multi-quantum well mode-locked SCOWL produced 10-ps pulses with energies of $58 \mathrm{pJ}$ and average powers of $250 \mathrm{~mW}$ at a repetition rate of 4.29 $\mathrm{GHz}$ achieving 5.8-W peak power [11]. In external-cavity configurations, record-high peak power pulses have been generated from edge-emitting external-cavity lasers with additional amplification and compression stages (and thus unavoidably larger footprint), resulting in pulses with $2.5 \mathrm{~kW}$ peak power [12]. VECSELs have also demonstrated their usefulness for ultrafast applications which require high peak power, such as multi-photon imaging [13]. Quantum-dots (QD) incorporated as the active medium in a diode laser offer, amongst other advantages, a broad gain bandwidth and ultrafast recovery time of the QD absorber making them ideal candidates for the generation of ultrashort pulses [1418]. For example, $Q D$ based saturable absorbers were effectively used for passively $\mathrm{Q}$-switched modelocking of diode pumped solid state lasers [19-20]. Theoretical and experimental investigations of 


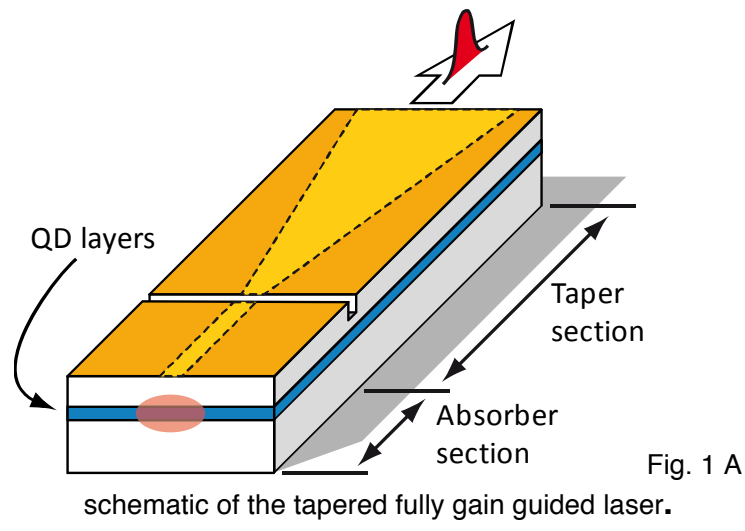

quantum-dot tapered lasers were undertaken [16,2122]. Tapered lasers can be designed in three different ways: fully index-guided, fully gain-guided or a mixture of both designs. The latter, as it was mentioned above, can generate high continuous-wave (CW) output power. Quantum-dot fully index-guided tapered lasers were able to generate transform-limited pulses with pulse durations of $360 \mathrm{fs}$, average power up to $15.6 \mathrm{~mW}$, resulting in only $2.25-\mathrm{W}$ peak power (under $12^{\circ} \mathrm{C}$ operating temperature) [23]. It was also previously shown that a reduction of the pulse duration from 2.3 ps to 800 fs can be achieved by changing the absorber-to-gain length ratio from 14:1 to 3:1 in straight QD waveguide lasers [24] and a similar approach has been successfully undertaken for two-section QD mode-locked index-guided tapered lasers $[16,22]$. On the other hand, fully gain-guided tapered lasers have the advantage of simpler, lower cost and rapid fabrication process compared to other tapered laser designs. A great improvement in output average power and peak power was shown in our previous work [25]. In that paper, the generation of 6ps pulses with high average power of $209 \mathrm{~mW}$ corresponding to a pulse energy of $14.2 \mathrm{pJ}$ was achieved. Furthermore, by using an absorber-to-gain length ratio of $1: 6$, high peak power of $3.6 \mathrm{~W}$ was directly generated from monolithic tapered fully gainguided 5-layer and 10-layer quantum-dot lasers [25]. In this present paper, we report record-high $17.7 \mathrm{~W}$ peak power from monolithic two-section quantum-dot tapered laser with 1.26 ps pulse duration. High average power of $287.7 \mathrm{~mW}$ with 2.2 ps pulses, corresponding to $28.7 \mathrm{pJ}$ pulse energy are achieved. Phase noise and integration timing jitter of $2.6 \mathrm{ps}$ is also demonstrated. Moreover, the generation of ultrashort Fourier-limited 672-fs pulses width with peak power of $3.4 \mathrm{~W}$ was achieved with another optimized structure where the absorber-to-gain length ratio was 5:1.

\section{Devices}

The QD wafer was grown on a $\mathrm{n}^{+}-\mathrm{GaAs}$ substrate by Molecular Beam Epitaxy (MBE), incorporating 10 identical layers of undoped InAs quantum dots (QDs) separated by 35-nm GaAs barriers which are used for eliminating high strain accumulation in each QD layer and integrated in an AIGaAs waveguide. The investigated fully gain-guided tapered lasers proposed and processed by III-V Lab consist of two separate and electrically-insulated sections: a narrow straight section near the back facet, which acts as a cavity spatial filter, extended by a tapered part which acts as an amplifier. Proton implantation has been performed outside the electrode area to achieve lateral gain guiding and to improve the electrical isolation between the two sections, resulting in a fully gain-guided twosection geometry. For mode-locked operation, a reverse bias and forward current are applied to the straight and tapered sections, which act as saturable absorber (SA) and gain sections, respectively. The two lasers with different designs are denoted with $A$ and $B$. The lengths of the straight and tapered sections are $0.4 \mathrm{~mm}(0.8 \mathrm{~mm})$ and $2.14 \mathrm{~mm}(3.2 \mathrm{~mm})$ for device $A(B)$ respectively resulting in a total cavity length of $2.54 \mathrm{~mm}(4.00 \mathrm{~mm})$, thus corresponding to a repetition rate of $16 \mathrm{GHz}(10 \mathrm{GHz})$. The full taper angle was $2^{\circ}$ for both devices with linear flares from $14 \mu \mathrm{m}(14 \mu \mathrm{m})$ to $88 \mu \mathrm{m}(124 \mu \mathrm{m})$. A schematic of the tapered fully gain-guided laser is presented in Fig.1. Anti- $\left(\mathrm{TiO}_{2} / \mathrm{SiO}_{2}\right)$ and high-reflective $\left(\mathrm{Al}_{2} \mathrm{O}_{3} / \mathrm{Si}\right)^{\star} 3$ coatings of $3 \%$ and $95 \%$ were deposited on the front/back facets respectively. The lasers were mounted on a Peltier cooler to maintain the operating temperature of $20^{\circ} \mathrm{C}$. Central emission wavelength was around $1260 \mathrm{~nm}$. The described designs have been derived from numerical simulations [26-28] of the mode-locking regimes and their dependence on the particular structure layout. In the following section, a brief description of the major findings from the design procedure is outlined.

\section{Design and numerical simulations}

The design of the optimized structures was obtained by performing preliminary beam propagation 


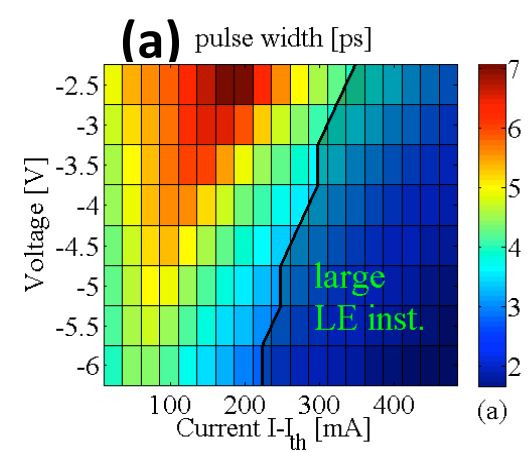

(c) (b) average power $[\mathrm{mW}]$

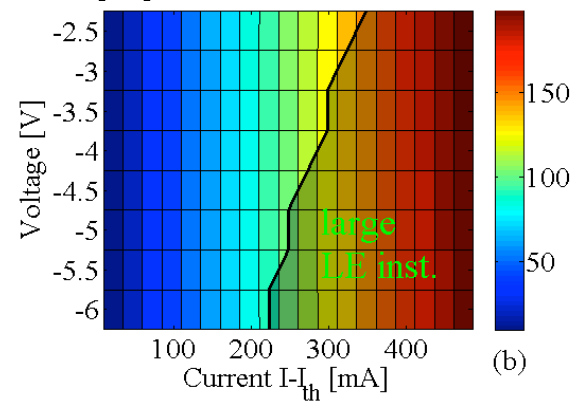

pulse peak power [mW]

Fig. 2 Simulated pulse width (a), average power

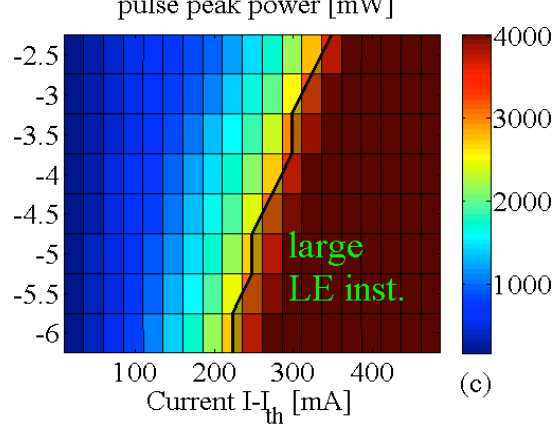
$\mathrm{SA}$ voltage and current above threshold $\mathrm{I}-\mathrm{I}_{\mathrm{th}}$, for a $4 \mathrm{~mm}$ device with a $2^{\circ}$ full angle tapered gain section and $17 \%$ SA section to gain section length ratio. Shaded region highlights unstable ML due to a large leading edge (LE) instability.
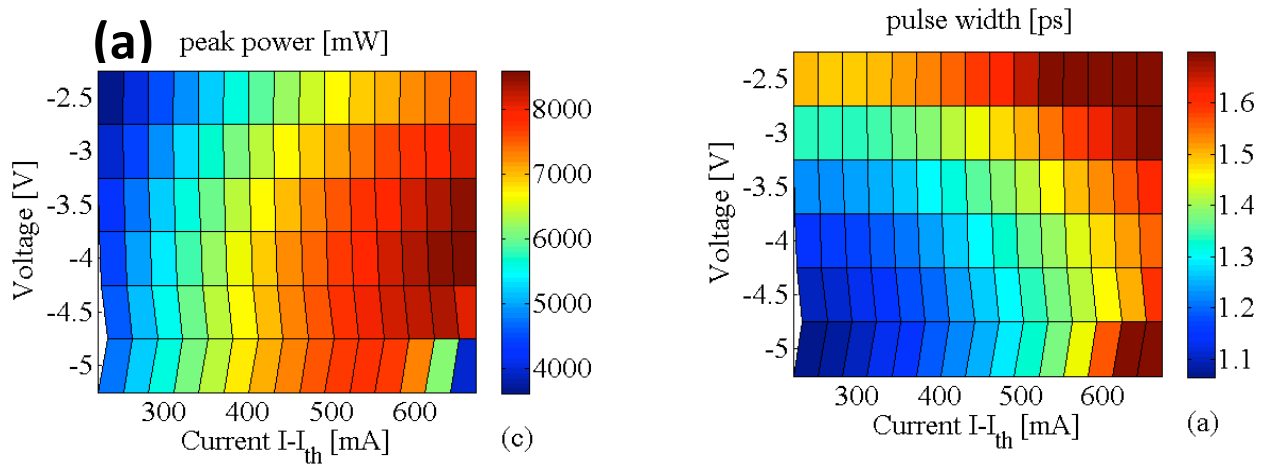

(C) average power $[\mathrm{mW}]$

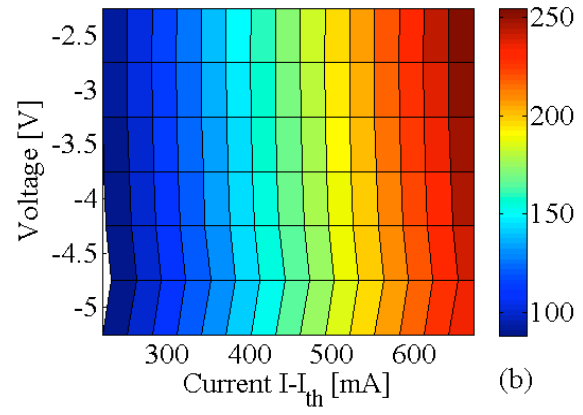

Fig. 3 Pulse width (a), average power (b) and peak power (c) as a function of the SA voltage and current above threshold I-I $\mathrm{I}_{\mathrm{th}}$, for a $4 \mathrm{~mm}$ device with $2^{\circ}$ full angle tapered gain section and $25 \%$ SA section to gain section length ratio. No leading edge instability to spontaneous emission noise perturbations has been observed in the investigated range of bias parameters. 


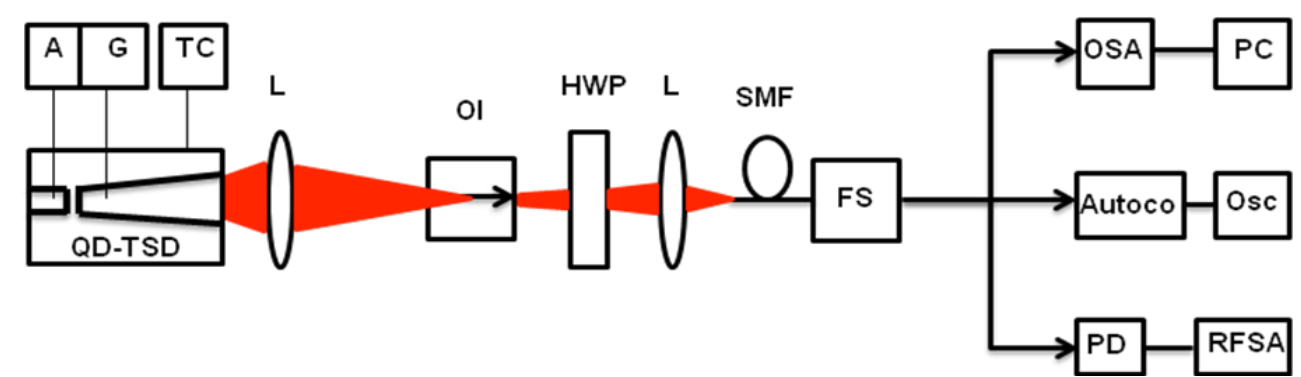

Fig. 4 Dynamic characterization experimental setup: QD -TSD: quantum dot - two section diode, A- absorber section, G: gain section, TC: temperature controller, L: lens, OI: optical isolator, HWP: half wave plate, SMF: single-mode fiber, FS: fiber splitter, OSA: optical spectrum analyzer; PC: personal computer; Autoco: autocorrelator; Osc: oscilloscope; PD: photo detector; RFSA: RF spectrum analyzer.

method simulations to verify the adiabatic transformation of the field in the tapered gain section, and by subsequently investigating the mode-locking regimes via both a delayed differential equation (DDE) numerical model [28] and a one-dimensional time-

to further optimize the gain guided device.

Long cavities with wide tapered sections have been successfully applied to enhance the output power in lasers operating in $\mathrm{CW}$ regime [6-8]. However, dynamic mode-locking simulations showed that long cavity passively mode-locked lasers tend to suffer a large leading edge instability with increasing current [29] induced by the spontaneous emission noise, ultimately limiting the maximum average and peak power achievable from such devices. In order to overcome this important limitation, two different approaches have been therefore pursued:

A) With respect to the device described in [25], we preserved a short cavity length $(2.5 \mathrm{~mm})$ but we

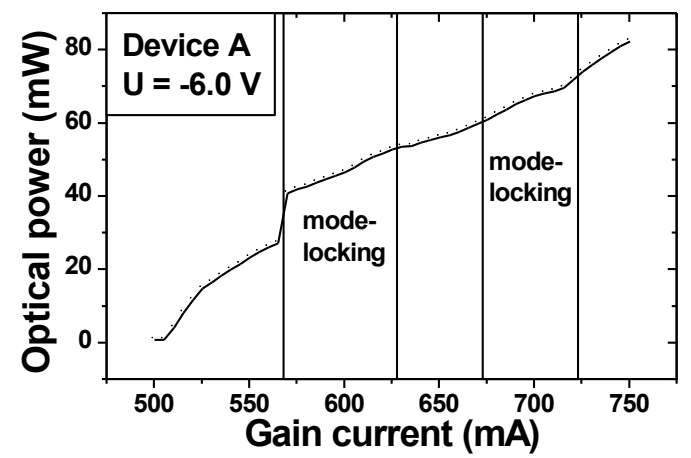

Fig. 5 Light current characteristic for device $A$ at a reverse bias of 6.0V. The shortest pulses are obtained at $570 \mathrm{~mA}$ and the highest peak power at $695 \mathrm{~mA}$. domain travelling-wave (TDTW) model [27], phenomenologically including the effect of the tapered gain section through a dependence of the active region volume and transverse field profile on the longitudinal position of the cavity.

Starting from the experimental results presented in [25], we investigated the possibility increased the SA section to gain section length ratio from 1:6 [25] to 1:5. As already pointed out in [23] and confirmed by our simulations, the increase in this ratio allows to significantly shorten the achieved modelocked pulses. As a matter of fact, the device operates in a condition where the QD ground state net gain is close to its maximum value due to the small available density of states and the high cavity losses; this ensures a very small differential gain that highly reduces the gain saturation due to total carrier density depletion. In addition, the tapered section with a large full angle of $2^{\circ}$ was considered allowing a further increase in the gain saturation energy. The ratio between the gain and absorption saturation energies is consequently highly improved [23] In this condition gain saturation balancing the absorption bleaching in the SA is mainly due to spectral hole burning nonlinearities enabling the generation of ultrashort modelocked pulses.

B) An alternative approach was then investigated. We considered a total device length $(4 \mathrm{~mm})$ much larger than in [25]. In order to avoid the onset of large leading edge instabilities with increasing current, a highly enhanced SA section to gain section length ratio was then considered. Simulations identified the ratio $1: 4$ as the optimum ratio. Furthermore the same full angle of $2^{\circ}$ characterizing the tapered gain section was considered. As shown in Fig. 2 and Fig. 3, 


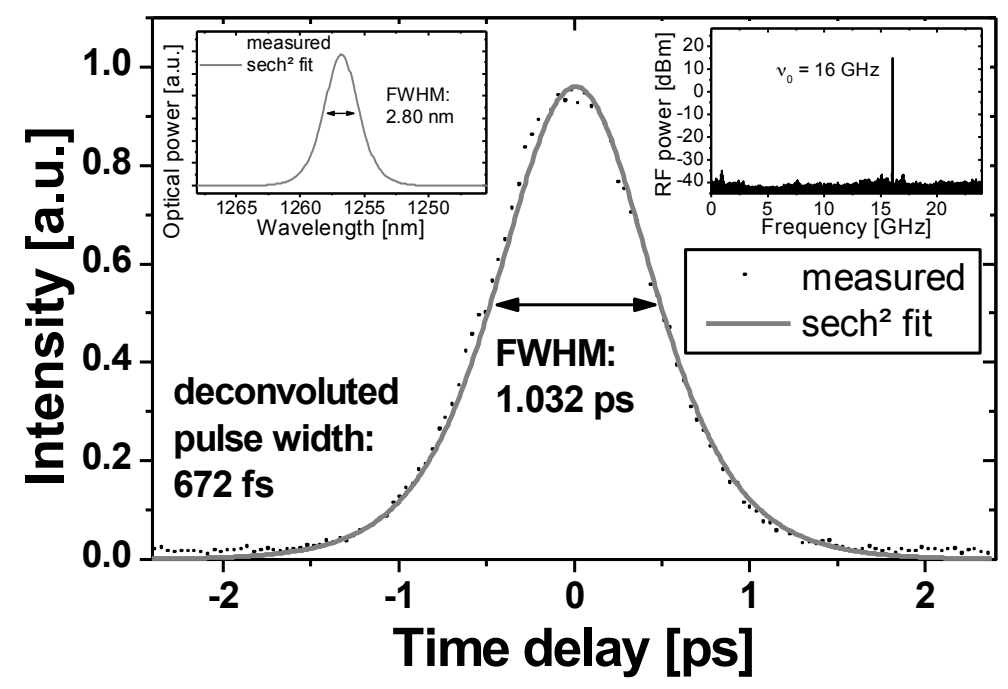

Fig. 6 Autocorrelation signal of the shortest pulse width of laser A; inset left: optical spectrum; inset right: RF spectrum at a gain current of $570 \mathrm{~mA}$ and a reverse bias of $-6.0 \mathrm{~V}$.

changing the SA section to gain section length ratio from 1:6 [25] to $1: 4$ allows to fully eliminate the onset of the leading edge instabilities in the ML regime, enabling large average power and, as for the case A, to significantly shorten the achieved mode-locked pulses. In addition, the reduced pulse repetition rate also contributes to the achievement of higher energy pulses.

\section{Experimental results and discussion}

Pulse measurements were carried out with an autocorrelator using second harmonic generation. Optical and RF spectra were recorded by respective analyzers. The experimental setup is shown in Fig. 4. We first identified the mode locking regime for device $A$ and selected the optimum reverse voltage for generation of the shortest pulses, which amounts to $6.0 \mathrm{~V}$. This results in a region of mode locking from $570 \mathrm{~mA}$ to $625 \mathrm{~mA}$ and from $675 \mathrm{~mA}$ to $720 \mathrm{~mA}$. Below $570 \mathrm{~mA}$ incomplete mode-locking occurs, above $720 \mathrm{~mA}$ strong modulations at a frequency of $560 \mathrm{MHz}$ are existent and in the intermediate non mode-locking region a progressive repetition rate transition takes place [30]. The light-current characteristic at $-6.0 \mathrm{~V}$ reverse bias is shown in Fig. 5 . The lasing threshold amounts to $500 \mathrm{~mA}$ at $-6.0 \mathrm{~V}$ and the total output power at a gain current of $720 \mathrm{~mA}$ amounts to $72 \mathrm{~mW}$. This current corresponds to the upper border of the ML regime and thus provides the highest average power. Mode locking starts at $570 \mathrm{~mA}$ which is close to lasing threshold. The shortest pulse width of $672 \mathrm{fs}$ is observed at $570 \mathrm{~mA}$ and the corresponding pulse trace is depicted in Fig.6. The calculated time-bandwidth-product amounts to 0.35 using a sech ${ }^{2}$ fit for the autocorrelation. Within the complete mode-locking regime a time-bandwidth product below 0.77 is measured and from $570 \mathrm{~mA}$ to $600 \mathrm{~mA}$ Fourier-limited pulses are obtained. The influence of the gain current on the pulse width is shown in Fig. 7. The pulse width increases from 672 fs at $570 \mathrm{~mA}$ to $938 \mathrm{fs}$ at $720 \mathrm{~mA}$.

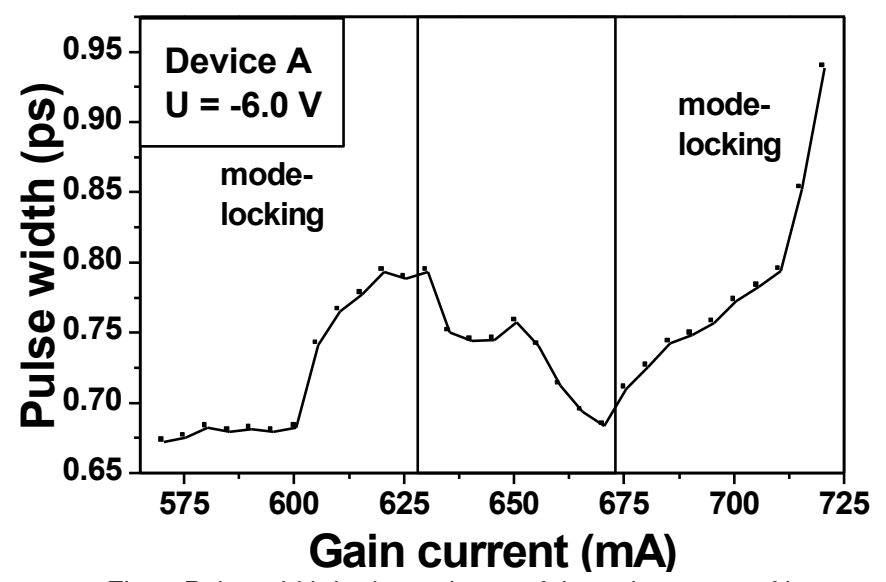

Fig. 7 Pulse width in dependence of the gain current of laser $\mathrm{A}$ at a reverse bias of $-6.0 \mathrm{~V}$.

We now estimate the peak power from the measured pulse width, average power and the repetition rate of $16 \mathrm{GHz}$. The peak power evolution in dependence of gain current is depicted in Fig. 8. Peak power increases from $3.8 \mathrm{~W}$ at $570 \mathrm{~mA}$ to $4.78 \mathrm{~W}$ at $720 \mathrm{~mA}$ with a maximum peak power of $5.48 \mathrm{~W}$ 


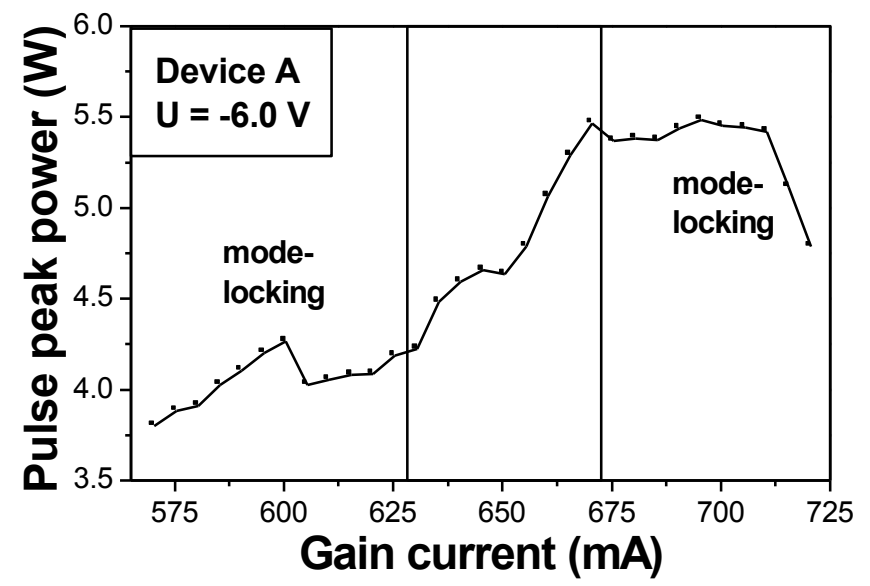

Fig. 8 Dependence of peak power as a function of the gain current of laser $A$, at a reverse bias of $-6.0 \mathrm{~V}$.

together with a pulse width of $757 \mathrm{fs}$ at a gain current of $695 \mathrm{~mA}$.

The measurements of the far field pattern on device $A$ presented in Fig. 9 are performed at $575 \mathrm{~mA}$ and 675 $\mathrm{mA}$ currents. We can notice the single mode behaviour together with a very low full width of $2.4^{\circ}$ and $3.1^{\circ}$ at $1 / \mathrm{e}^{2}$ of maximum respectively. $\mathrm{M}^{2}$ values are 1.3 and 1.7 , for the gain current values of $575 \mathrm{~mA}$ and $675 \mathrm{~mA}$, respectively (reverse bias of $-6 \mathrm{~V}$ ).

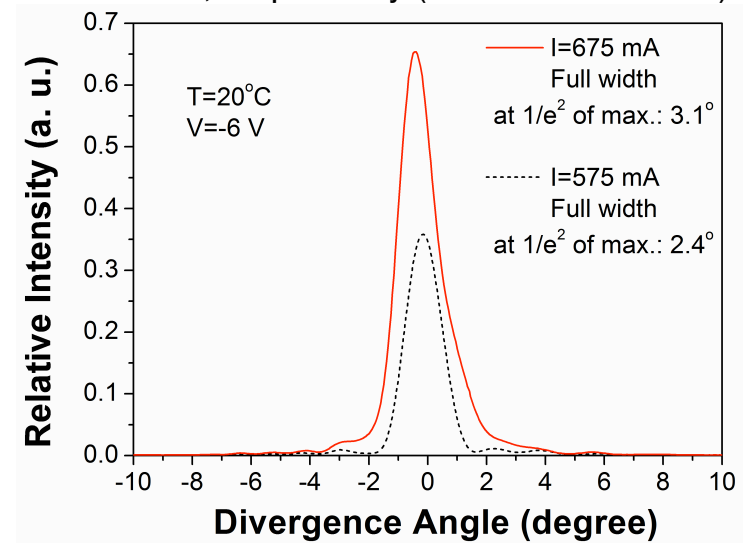

Fig. 9 Far field slow-axis pattern measured on device $A$ at $575 \mathrm{~mA}$ and $675 \mathrm{~mA}$ with a very low full width of $2.4^{\circ}$ and $3.1^{\circ}$ at $1 / \mathrm{e}^{2}$ of maximum respectively.

As indicated by numerical simulations, an alternative way to even further increase peak power is increasing the overall laser length and the absorber- to-gain-length-ratio as realized in device $\mathrm{B}$, where stable mode-locking was achieved for reverse bias between $3 \mathrm{~V}$ and $6 \mathrm{~V}$ applied to the absorber and gain current between $800 \mathrm{~mA}$ and $1800 \mathrm{~mA}$. Indeed, the optimum mode locking regime for device $B$ is found at a reverse bias of $-4.0 \mathrm{~V}$, attributed to the change in absorber-to-gain-length-ratio. In analogy to Fig. 5 the average power dependence of gain current under uniform injection and under an applied reverse bias of $-4.0 \mathrm{~V}$ are shown in Fig. 10a, b.
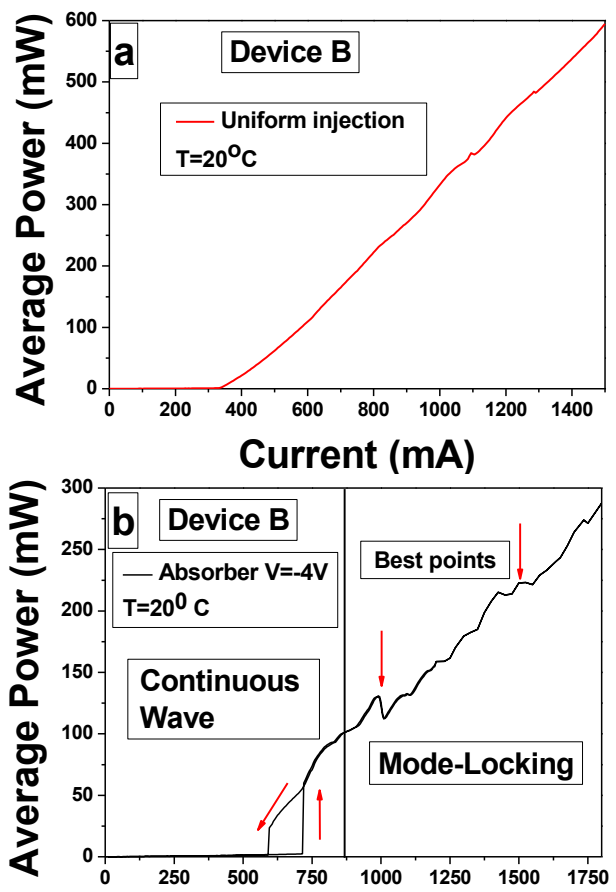

10 Lightcurrent curves for laser $B$ at Current (mA) $20^{\circ}$ C a) under uniform injection (red line) with a threshold current of $341 \mathrm{~mA}$ and $b$ ) with $-4 \mathrm{~V}$ on the absorber section (black line) with $720 \mathrm{~mA}$ threshold current.

Under uniform injection a threshold current of $341 \mathrm{~mA}$ and differential efficiency of 0.38 W/A were observed. When a reverse bias of $-4 \mathrm{~V}$ is applied on the absorber, laser emission takes place at $720 \mathrm{~mA}$ with a hysteresis loop. Self starting mode-locking regime was observed from current of $830 \mathrm{~mA}$ up to 1.7 A. Compared to laser $A$, the threshold is shifted from $500 \mathrm{~mA}$ to $720 \mathrm{~mA}$ and the start of the mode locking region is shifted from $575 \mathrm{~mA}$ to $830 \mathrm{~mA}$. Both 
changes confirm our assumption of the determining influence of the ratio of gain-to-absorber lengths. The maximum output average power achieved was 287.7 $\mathrm{mW}$, for a reverse bias of $-4 \mathrm{~V}$ and current $1.8 \mathrm{~A}$ at 20 ${ }^{\circ} \mathrm{C}$, corresponding to $28.7 \mathrm{pJ}$ pulse energy with $2.2 \mathrm{ps}$ pulse duration (Fig. 10b). This corresponds to an increase in average power by a factor of 4.5 as compared to laser A as reflected directly as one contribution to the peak power. Pulse duration increases from 820 fs to 2.2 ps with increasing current and is only slightly higher than compared to the pulse observed with laser A, as shown in Fig.11a. Peak power varies between $9 \mathrm{~W}$ and $17.7 \mathrm{~W}$ depending on the bias conditions (Fig 11b).
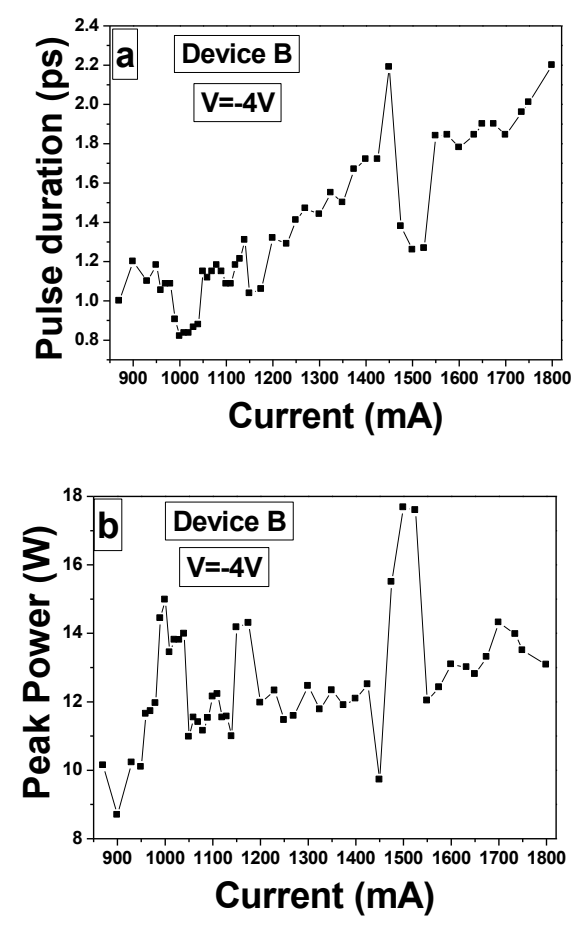

Fig. 11a) Pulse duration and b) peak power dynamics at 4-V reverse bias for quantum-dot tapered laser $B$.

The shortest pulse width of 820 fs for a reverse bias of $-4 \mathrm{~V}$ and injected current of $1 \mathrm{~A}$ was obtained associated at the same time with high peak power of $15 \mathrm{~W}$ and a high average power of $123 \mathrm{~mW}$ and, which is made possible due to the increasing length of the tapered section and the reduction of the repetition rate by a factor of 0.62 (Fig 12a, b). The optical spectrum was centred at $1259.5 \mathrm{~nm}$ with a full-width half-maximum of $5.36 \mathrm{~nm}$, resulting in a timebandwidth product of 0.83 . 

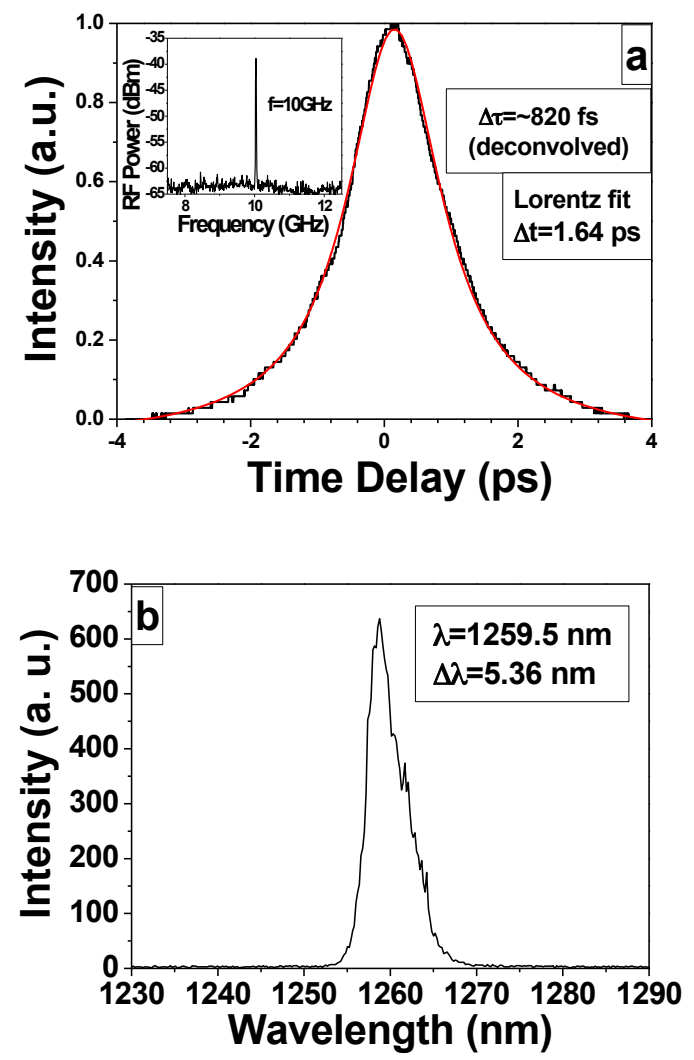

Fig. 12a) Autocorrelation (Inset: RF spectrum); b) optical spectrum for an injection current of $1 \mathrm{~A}$ and reverse bias $-4 \mathrm{~V}$ for short pulse regime for quantum-dot tapered laser $B$.

A record-high peak power of $17.7 \mathrm{~W}$ with 1.26 ps pulse duration and $222.7 \mathrm{~mW}$ average power was attained for $-4 \mathrm{~V}$ and $1.5 \mathrm{~A}$ bias condition (Fig. $13 \mathrm{a}, \mathrm{b}$ ). This represents the highest reported peak power to be generated from mode-locked monolithic semiconductor lasers. It is noteworthy to mention that the central wavelength suffered a nearly negligible shift to $1260.4 \mathrm{~nm}$ for such a high current of $1.5 \mathrm{~A}$ (resulting in current density only of $671 \mathrm{~A} / \mathrm{cm}^{2}$ ), suggesting that the band filling effect is quite weak for this device. The full-width at half-maximum of the optical spectrum was $5.6 \mathrm{~nm}$, resulting in a timebandwidth product of 1.33 . Mode-locking was also observed for reverse bias between $-2 \mathrm{~V}$ and $-6 \mathrm{~V}$. Compared to the already significant peak power achieved by laser $A$, we have furthermore successfully improved the peak power by a factor of 3.8 to record values by a combined increase of the 

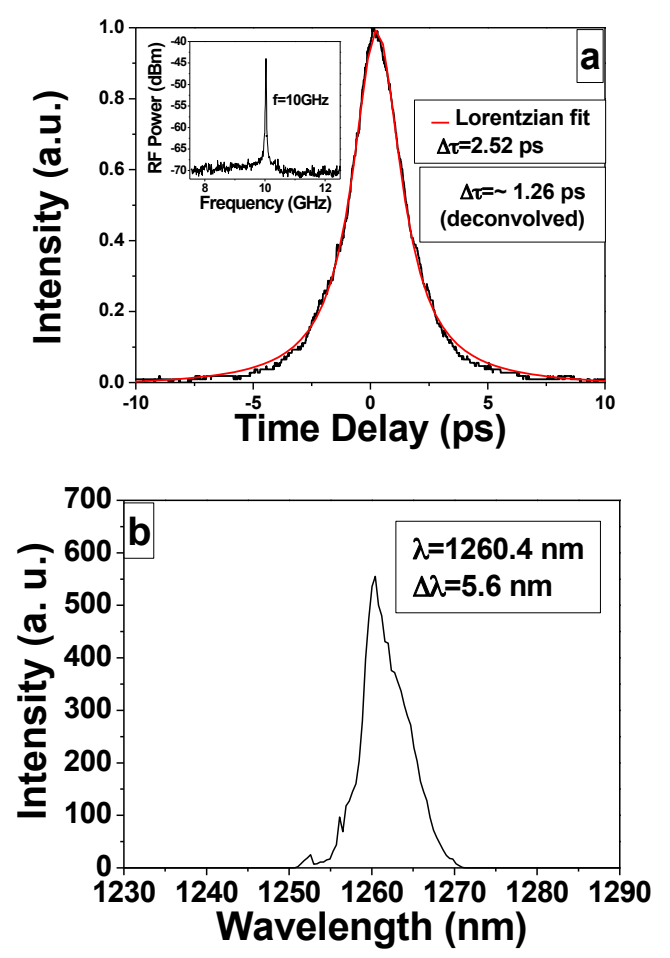

Fig. 13a) Autocorrelation (Inset: RF spectrum); b) optical spectrum for an injection current of $1.5 \mathrm{~A}$ and reverse bias $-4 \mathrm{~V}$ for record-high peak power regime for quantum-dot tapered laser $B$.

total laser length by a factor of 1.6 and the absorberto-gain-ratio by a factor of 1.25 . Far-field pattern was also measured on device $B$ at $1 \mathrm{~A}$ (the shortest pulse regime) and $1.5 \mathrm{~A}$ (the highest peak power regime).

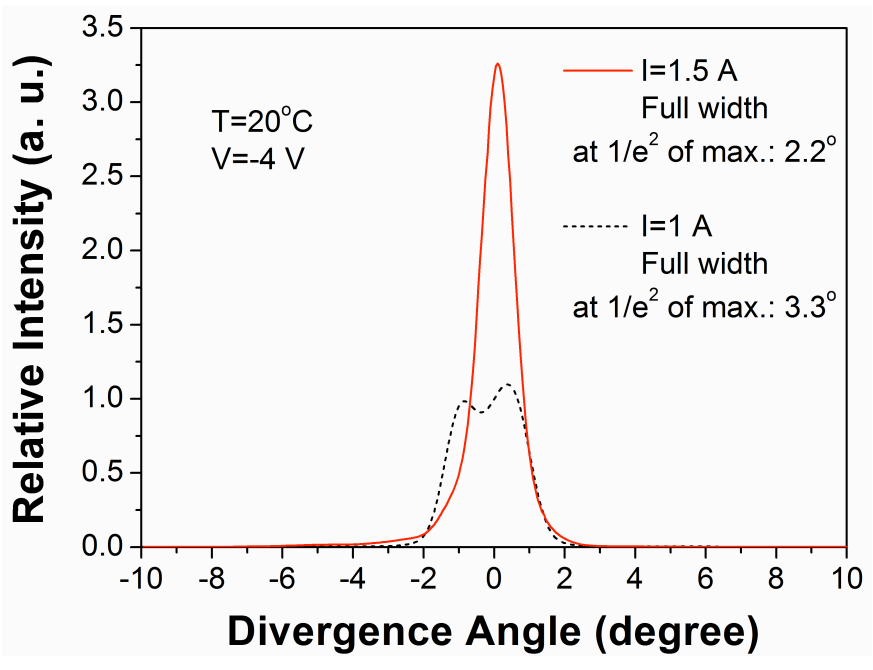

Fig. 14 Shows far field slow-axis pattern measured on device $\mathrm{B}$ at $1 \mathrm{~A}$ (shortest pulse width) and 1.5A (highest peak power) with very low full width of $3.3^{\circ}$ and $2.2^{\circ}$ at $1 / \mathrm{e}^{2}$ of maximum respectively.

A single mode behaviour was exhibited at $1.5 \mathrm{~A}$ with very low full width of $2.2^{\circ}$ at $1 / \mathrm{e}^{2}$ of maximum. We also observed two lobes behaviour at $1 \mathrm{~A}$ and full width of $3.3^{\circ}$ at $1 / \mathrm{e}^{2}$ of maximum which corresponds to single high-order lateral mode (Fig. 14). $\mathrm{M}^{2}$ values are 2.9 and 2.1 at gain current of $1 \mathrm{~A}$ and $1.5 \mathrm{~A}$ at bias of $-4 \mathrm{~V}$ respectively.

Phase noise measurements show that the integrated timing jitter for laser $B$ can be as low as 2.6 ps (from $10 \mathrm{~Hz}$ to $50 \mathrm{MHz}$ ) at $14^{\circ} \mathrm{C}$ (Fig. 15). This result shows that mode-locked QD two-section gain-guided tapered lasers are not only useful for the generation of ultrashort pulses with high peak power, but they are also promising as sources for low noise applications.

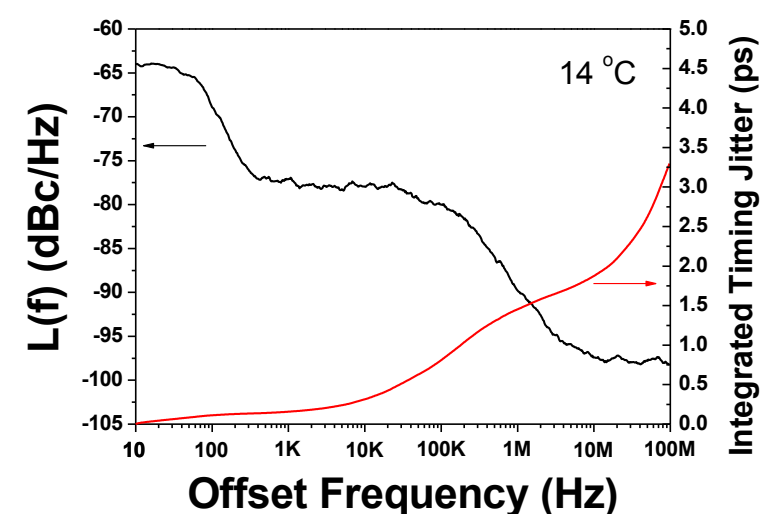

Fig. 15 Phase noise and integrated timing jitter for an injection current of $955 \mathrm{~mA}$ and reverse bias of $-4.17 \mathrm{~V}$ at $14^{\circ} \mathrm{C}$ for laser $\mathrm{B}$. 


\section{Conclusions}

Passively mode-locked quantum-dot gain-guided tapered lasers with different structural parameters have been simulated, fabricated and investigated. Optimized structure designs were obtained by numerical simulations and the experimental findings fully confirm the trends identified by the modelling activity. Record high peak power and sub-picosecond Fourier-limited pulse generation have been demonstrated by two novel passively mode-locked fully gain-guided two-section quantum dot lasers with tapered gain sections. A peak power of $17.7 \mathrm{~W}$ with a pulse width of $1.26 \mathrm{ps}$ and peak power of $3.8 \mathrm{~W}$ with a Fourier-limited pulse width of $672 \mathrm{fs}$ is achieved by a proper choice of active region and laser structure geometry including absorber-to-gain ratio and total laser length. Quantum-dot tapered gain-guided lasers have therefore shown promising results as high power ultra-fast and ultra-compact semiconductor-based laser sources. In future work, we plan to perform frequency-resolved optical gating (FROG) measurements, such as reported in [31-33].

\section{Acknowledgement}

This work was supported by the EU FP7 (FAST-DOT project Grant No. 224338). M. A. C. also acknowledges support via a Royal Academy of Engineering/EPSRC Research Fellowship. The authors would also like to thank Eric Vinet for excellent technical assistance.

\section{Authors contributions}

All the groups and authors have contributed equally to the scientific results presented in this paper by experiments (UoD \& TUD), modelling (PoliTo) and technology (Innolume \& III-V Lab).

\section{References}

[1] Masaru Kuramoto, Nobuyoshi Kitajima, Hengchang Guo, Yuji Furushima, Masao Ikeda, and Hiroyuki Yokoyama, Opt. Lett. 32, 2726 (2007).
[2] Tomoko Shimada, Wataru Watanabe, Sachihiro Matsunaga, Tsunehito Higashi, Hiroshi Ishii, Kiichi Fukui, Keisuke Isobe, and Kazuyoshi Itoh, Opt. Express 13, 9869 (2005).

[3] Rafael R. Gattass and Eric Mazur, Nat Photon 2, 219 (2008).

[4] G. Hollemann, B. Braun, P. Heist, J. Symanowski, U. Krause, J. Kraenert, and C. Deter, Proceedings of SPIE 4294, 36 (2001).

[5] M. Attygalle, A. Nirmalathas, and H. F. Liu, Optics Communications 217, 161 (2003).

[6] K. Paschke, B. Sumpf, F. Dittmar, G. Erbert, R. Staske, H. Wenzel, and G. Trankle, Selected Topics in Quantum Electronics, IEEE Journal of 11, 1223 (2005).

[7] M. T. Kelemen, J. Weber, G. Kaufel, G. Bihlmann, R. Moritz, M. Mikulla, and G. Weimann, Electronics Letters 41, 1011 (2005).

[8] B. Sumpf, K. H. Hasler, P. Adamiec, F. Bugge, F. Dittmar, J. Fricke, H. Wenzel, M. Zorn, G. Erbert, and G. Trankle, Selected Topics in Quantum Electronics, IEEE Journal of 15, 1009 (2009).

[9] Alan Mar, Roger Helkey, W. X. Zou, D. Bruce Young, and John E. Bowers, Applied Physics Letters 66, 3558 (1995).

[10] K. G. Wilcox, A. H. Quarterman, H. Beere, D. A. Ritchie, and A. C. Tropper, Photonics Technology Letters, IEEE 22, 1021 (2010).

[11] Jason J. Plant, Juliet T. Gopinath, Bien Chann, Daniel J. Ripin, Robin K. Huang, and Paul W. Juodawlkis, Opt. Lett. 31, 223 (2006).

[12] T. Schlauch, M. Li, M. R. Hofmann, A. Klehr, G. Erbert, and G. Trankle, Electronics Letters 44, 678 (2008).

[13] Rodrigo Aviles-Espinosa, George Filippidis, Craig Hamilton, Graeme Malcolm, Kurt J. Weingarten, Thomas Südmeyer, Yohan Barbarin, Ursula Keller, Susana I. C. O. Santos, David Artigas, and Pablo Loza-Alvarez, Biomed. Opt. Express 2, 739 (2011).

[14] E. U. Rafailov, M. A. Cataluna, and W. Sibbett, Nat Photon 1, 395 (2007). 
[15] M. Kuntz, G. Fiol, M. Laemmlin, C. Meuer, and D. Bimberg, Proceedings of the IEEE 95, 1767 (2007).

[16] M. G. Thompson, A. R. Rae, Xia Mo, R. V. Penty, and I. H. White, Selected Topics in Quantum Electronics, IEEE 15, 661 (2009).

[17] M. A. Cataluna, Y. Ding, D. I. Nikitichev, K. A. Fedorova, and E. U. Rafailov, Selected Topics in Quantum Electronics, IEEE PP, 1 (2011).

[18] Edik U. Rafailov, Maria Ana Cataluna, and Eugene A. Avrutin, Ultrafast lasers based on quantum-dot structures: physics and devices. (Wiley-VCH, 2011).

[19] M. S. Gaponenko, V. E. Kisel, N. V. Kuleshov, A. M. Malyarevich, K. V. Yumashev, and A. A. Onushchenko, Laser Physics Letters 7, 286 (2010).

[20] G. Li, B. Q. Yao, P. B. Meng, W. Wang, Y. L. Ju, and Y. Z. Wang, Laser Physics Letters 8, 42 (2011).

[21] W. Kaiser, J. P. Reithmaier, A. Forchel, H. Odriozola, and I. Esquivias, Applied Physics Letters 91, 051126 (2007).

[22] M. G. Thompson, A. Rae, R. L. Sellin, C. Marinelli, R. V. Penty, I. H. White, A. R. Kovsh, S. S. Mikhrin, D. A. Livshits, and I. L. Krestnikov, Applied Physics Letters 88, 133119 (2006).

[23] M. G. Thompson, A. R. Rae, Xia Mo, R. V. Penty, and I. H. White, Selected Topics in Quantum Electronics, IEEE Journal of 15, 661 (2009).

[24] A. R. Rae, M. G. Thompson, A. R. Kovsh, R. V. Penty, and I. H. White, Photonics Technology Letters, IEEE 21, 307 (2009).
[25] D. Nikitichev, Y. Ding, M. Ruiz, M. Calligaro, N. Michel, M. Krakowski, I. Krestnikov, D. Livshits, M. Cataluna, and E. Rafailov, Applied Physics B 103, 609 (2010).

[26] M. Rossetti, P. Bardella, M. Gioannini, and I. Montrosset, in European conference on Lasers and Electro-Optics 2009 and the European Quantum Electronics Conference. CLEO Europe - EQEC 2009. (Conference Digest (Optical Society of America, 2009), 2009), p. paper CF_P21.

[27] M. Rossetti, P. Bardella, and I. Montrosset, Quantum Electronics, IEEE Journal of 47, 139 (2011).

[28] M. Rossetti, P. Bardella, and I. Montrosset, Quantum Electronics, IEEE Journal of 47, 569 (2011).

[29] A. G. Vladimirov and et al., Phys. Rev. A 72, 033808 (2005).

[30] K. Merghem, A. Akrout, A. Martinez, G. Moreau, J. P. Tourrenc, F. Lelarge, F. Van Dijk, G. H. Duan, G. Aubin, and A. Ramdane, Opt. Express 16, 10675 (2008).

[31] L. Lester, D. Kane, N. Usechak, C. Lin, Y. Li, Y. Xin, and V. Kovanis, Proceedings of SPIE 7616 (2010).

[32] Y. C. Xin, L. F. Lester, and D. Kane, Electronics Letters 44, 1255 (2008).

[33] H. Schmeckebier, G. Fiol, C. Meuer, D. Arsenijevi, and D. Bimberg, Opt. Express 18, 3415 (2010). 